\title{
215.
}

\section{A SUPPLEMENTARY MEMOIR ON THE PROBLEM OF DISTURBED ELLIPTIC MOTION.}

[From the Memoirs of the Royal Astronomical Society, vol. xxviII. pp. 217-234. Read January 12, 1860.]

THE present memoir contains an investigation upon fundamentally different principles of a system of formulæ given in my "Memoir on the Problem of Disturbed Elliptic Motion," Ast. Soc. Mem. t. xxvII. pp. 1-29 (1858), [212]; it is shown how the formulæ are deduced by means of the general equations of the Mécanique Analytique, from the expression for the Vis viva function $T$, in terms of the coordinates ( $r$ the radius vector, $p$ the departure) of the body in its instantaneous orbit, viz., the ultimate form of this function is $T d t^{2}=\frac{1}{2}\left(d r^{2}+r^{2} d p^{2}\right)$, but $T$ contains in the first instance terms, not identically vanishing, but which are to be equated to zero, thus furnishing equations of the problem which could not be obtained from the foregoing ultimate form of $T$. The investigation throws, I think, a further light upon the system of formulæ, and completes the development which I was anxious to give of the dynamical problem. I have been a great deal indebted, in the composition of the memoir, to a correspondence some time ago with Professor Donkin on the general subject.

The word orbit is used to denote a great circle of the sphere, and it is assumed that in any orbit there is an origin of longitudes; the angular position of a body in reference to the orbit is determined by the longitude and latitude. It is ultimately assumed that the longitude is measured from an origin which is what I have called a departure-point; viz., in the general case of a variable orbit the departure-point is the point of intersection of the orbit by any orthogonal trajectory of the successive positions of the orbit: this includes the ease of a fixed orbit, where the departurepoint is simply a fixed point. As regards points in the orbit, the word departure may be used instead of longitude. In the present memoir the origin is in the first instance taken to be, not a departure-point, but an arbitrarily varying point of the orbit. 
The mutual position of any two orbits whatever, say the position of an orbit $x^{\prime} y^{\prime}$ and of the origin $x^{\prime}$ therein, in reference to the orbit $x y$ and the origin $x$ therein, is determined by

$$
\begin{aligned}
& \theta, \text { the longitude of node, } \\
& \sigma \text {, the departure of node, } \\
& \phi, \text { the inclination, }
\end{aligned}
$$

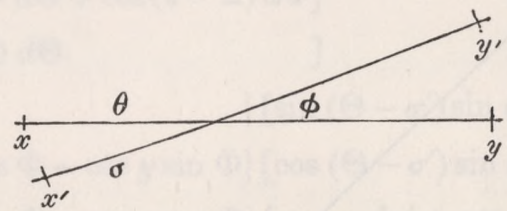

where the expression departure of node is used by way of distinction from longitude of node, the departure referring to the orbit $x^{\prime} y^{\prime}$ and origin $x^{\prime}$, the positions of which are to be determined, and the longitude to the orbit of reference $x y$ and origin $x$. And this distinction will be preserved throughout. It should be recollected that it is not as yet assumed that the origins are departure-points.

Consider now a fixed orbit $x_{0} y_{0}$ and fixed origin $x_{0}$ therein, and suppose that the orbit $x_{1} y_{1}$ and origin $x_{1}$ therein are determined in reference to the orbit $x_{0} y_{0}$ and origin $x_{0}$ therein, by

$$
\begin{aligned}
& \theta^{\prime}, \text { the longitude of node, } \\
& \sigma^{\prime} \text {, the departure of node, } \\
& \phi^{\prime} \text {, the inclination, }
\end{aligned}
$$

the orbit $x_{2} y_{2}$ and origin $x_{2}$ therein, in reference to the orbit $x_{1} y_{1}$ and origin $x_{1}$ therein, by
$\Theta$, the longitude of node,
$\Sigma$, the departure of node,
$\Phi$, the inclination,

and, finally, the position of the body in reference to the orbit $x_{2} y_{2}$ and origin $x_{2}$ therein, by

$$
\begin{aligned}
& r \text {, the radius vector, } \\
& v \text {, the longitude, } \\
& y \text {, the latitude. }
\end{aligned}
$$

It is required to find the expression of $T$, the Vis viva function, or half square of the velocity. We may imagine the rectangular axes $x_{0} y_{0} z_{0}, x_{1} y_{1} z_{1}, x_{2} y_{2} z_{2}$, the positions of which are determined as above, and the rectangular axes $x_{3} y_{3} z_{3}$, that of $x_{3}$ passing through the body, that of $y_{3}$ lying in the orbit $x_{2} y_{2}, 90^{\circ}$ in advance of the body or 
passing through the pole of the latitude circle, and that of $z_{3}$ in the latitude circle $90^{\circ}$ above the body. Or considering $x_{3}, y_{3}, z_{3}$, as points of the sphere, their positions in reference to the orbit $x_{2} y_{2}$ and origin $x_{2}$ therein are determined as follows, viz.,

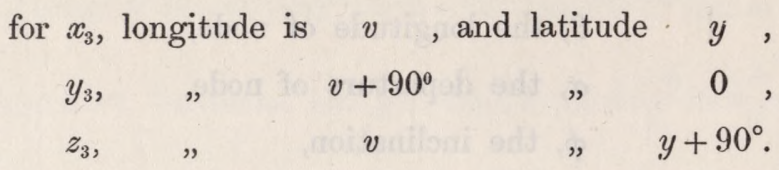

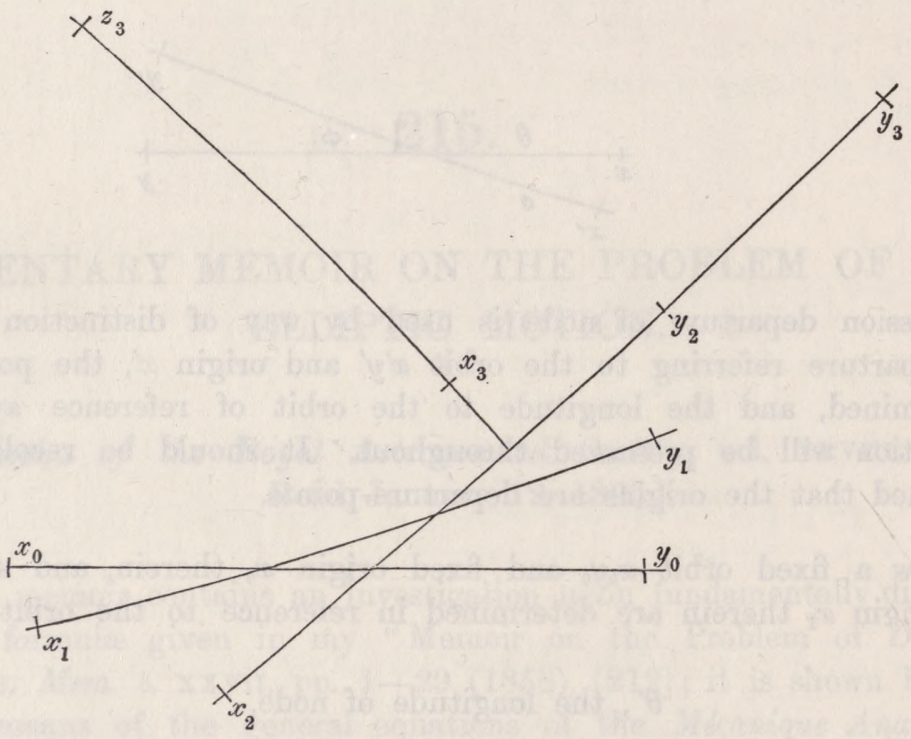

and we may suppose that $\left(x_{0}, y_{0}, z_{0}\right),\left(x_{1}, y_{1}, z_{1}\right),\left(x_{2}, y_{2}, z_{2}\right),\left(x_{3}, y_{3}, z_{3}\right)$, are the coordinates of any point in reference to these sets of axes respectively, the coordinates of the body in reference to the axes $x_{3} y_{3} z_{3}$ being obviously $(r, 0,0)$.

The displacernents in the directions of the axes $x_{3} y_{3} z_{3}$ respectively of a point the coordinates of which are $x_{3}, y_{3}, z_{3}$, are as usual

$$
\begin{aligned}
& d x_{3}+\left(-R y_{3}+Q z_{3}\right) d t, \\
& d y_{3}+\left(-P z_{3}+R x_{3}\right) d t, \\
& d z_{3}+\left(-Q x_{3}+P y_{3}\right) d t
\end{aligned}
$$

where the expressions of the rotations $P, Q, R$ are as follows, viz.,

$$
\begin{aligned}
P d t= & \sin y d v \\
& +\cos y[\sin (v-\Sigma) \sin \Phi d \Theta+\cos (v-\Sigma) d \Phi] \\
& +\sin y[-d \Sigma+\cos \Phi d \Theta \quad] \\
& +\{\cos y \cos (v-\Sigma) \\
& +\{\cos y \sin (v-\Sigma) \cos \Phi-\sin y \sin \Phi\}\left[\cos \left(\Theta-\sigma^{\prime}\right) \sin \phi^{\prime} d \theta^{\prime} \cos \left(\Theta-\sigma^{\prime}\right) d \phi^{\prime}\right] \\
& +\left\{\cos y \sin (v-\Sigma) \sin \left(\Theta-\sigma^{\prime}\right) d \phi^{\prime}\right] \\
& \text { sin } y \cos \Phi\}\left[-d \sigma^{\prime}+\cos \phi^{\prime} d \theta^{\prime}\right.
\end{aligned}
$$




$$
\begin{aligned}
& Q d t=-d y \\
& +[\cos (v-\Sigma) \sin \Phi d \Theta-\sin (v-\Sigma) d \Phi] \\
& -\sin (v-\Sigma)\left[\sin \left(\Theta-\sigma^{\prime}\right) \sin \phi^{\prime} d \theta^{\prime}+\cos \left(\Theta-\sigma^{\prime}\right) d \phi^{\prime}\right] \\
& +\cos (v-\Sigma) \cos \Phi\left[\cos \left(\Theta-\sigma^{\prime}\right) \sin \phi^{\prime} d \theta^{\prime}-\sin \left(\Theta-\sigma^{\prime}\right) d \phi^{\prime}\right] \\
& +\cos (v-\Sigma) \sin \Phi\left[\quad-d \sigma^{\prime}+\cos \phi^{\prime} d \theta^{\prime}\right.
\end{aligned}
$$

$R d t=\cos y d v$

$-\sin y[\sin (v-\Sigma) \sin \Phi d \Theta+\cos (v-\Sigma) d \Phi]$

$+\cos y[-d \Sigma+\cos \Phi d \Theta$

$+\{-\sin y \cos (v-\Sigma)$

\}$\left[\sin \left(\Theta-\sigma^{\prime}\right) \sin \phi^{\prime} d \theta^{\prime}+\cos \left(\Theta-\sigma^{\prime}\right) d \phi^{\prime}\right]$

$+\{-\sin y \sin (v-\Sigma) \cos \Phi-\cos y \sin \Phi\}\left[\cos \left(\Theta-\sigma^{\prime}\right) \sin \phi^{\prime} d \theta^{\prime}-\sin \left(\Theta-\sigma^{\prime}\right) d \phi^{\prime}\right]$

$+\{-\sin y \sin (v-\Sigma) \sin \Phi+\cos y \cos \Phi\}\left[\quad-d \sigma^{\prime}+\cos \phi^{\prime} d \theta^{\prime}\right.$

For the body itself we must write $r, 0,0$, in the place of $x_{3}, y_{3}, z_{3}$; the displacements are therefore

$$
d r, \quad r R d t, \quad-r Q d t
$$

and the expression for the Vis viva function is

$$
T d t^{2}=\frac{1}{2}\left\{d r^{2}+r^{2}\left(Q^{2}+R^{2}\right) d t^{2}\right\}
$$

where $Q, R$ have the above-mentioned values.

Let $\Omega$ be a function of the coordinates $x_{0}, y_{0}, z_{0}$ of a point, $\Omega$ will be a function of $x_{3}, y_{3}, z_{3}$, and also of the quantities $\Theta, \Sigma, \Phi, \theta^{\prime}, \sigma^{\prime}, \phi^{\prime}$ which determine the positions of the axes $x_{3}, y_{3}, z_{3}$, and the complete differential of $\Omega$ will be

$$
\begin{aligned}
d \Omega= & \frac{d \Omega}{d x_{3}}\left\{d x_{3}+\left(-R y_{3}+Q z_{3}\right) d t\right\} \\
& +\frac{d \Omega}{d y_{3}}\left\{d y_{3}+\left(-P z_{3}+{ }_{3} R x_{3}\right) d t\right\} \\
& +\frac{d \Omega}{d z_{3}}\left\{d z_{3}+\left(-Q x_{3}+P y_{3}\right) d t\right\}
\end{aligned}
$$

I assume for the present the truth of the following proposition, viz., that when $\Omega$ is a function of the coordinates of the body (in which case, as before, the values of $x_{3}, y_{3}, z_{3}$ are $\left.r, 0,0\right)$, we have

$$
\begin{aligned}
& \frac{d \Omega}{d x_{3}}=\quad \frac{d \Omega}{d r}, \\
& \frac{d \Omega}{d y_{3}}=\frac{1}{r} \sec y \frac{d \Omega}{d v}, \\
& \frac{d \Omega}{d z_{3}}=\quad \frac{1}{r} \frac{d \Omega}{d y} .
\end{aligned}
$$


$\Omega$ is here a function of $r, v, y, \Theta, \Sigma, \Phi, \theta^{\prime}, \sigma^{\prime}, \phi^{\prime}$, or, as we may express it, $\Omega=\Omega\left(r, v, y, \Theta, \Sigma, \Phi, \theta^{\prime}, \sigma^{\prime}, \phi^{\prime}\right)$; and the expression for the complete differential, by what precedes, is

$$
d \Omega=\frac{d \Omega}{d r} d r+R d t . \sec y \frac{d \Omega}{d v}-Q d t \frac{d \Omega}{d y}
$$

or, substituting for $Q, R$, their values, the expression is

$$
d \Omega=\frac{d \Omega}{d r} d r
$$$$
+\sec y \frac{d \Omega}{d v}\left\{\begin{array}{l}
\cos y d v \\
-\sin y[\sin (v-\Sigma) \sin \Phi d \Theta+\cos (v-\Sigma) d \Phi] \\
+\cos y[-d \Sigma+\cos \Phi d \Theta \\
+\{-\sin y \cos (v-\Sigma) \\
+\{-\sin y \sin (v-\Sigma) \cos \Phi-\cos y \sin \Phi\}\left[\cos \left(\Theta-\sigma^{\prime}\right) \sin \phi^{\prime} d \theta^{\prime}-\sin \left(\Theta-\sigma^{\prime}\right) d \phi^{\prime}\right] \\
+\{-\sin y \sin (v-\Sigma) \sin \Phi+\cos y \cos \Phi\}\left[\quad-d \sigma^{\prime}+\cos \phi^{\prime} d \theta^{\prime}\right.
\end{array}\right\}
$$

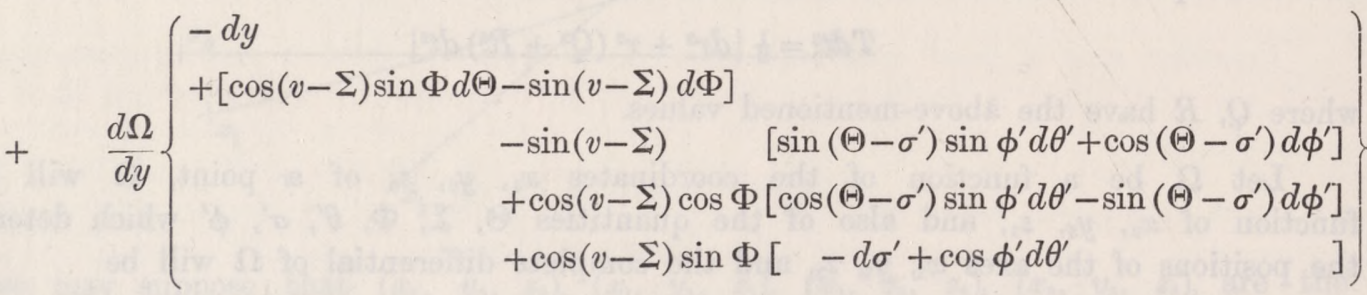

where, on the right-hand side, the terms involving the differentials of $r, v, y$, are, as they should be, $\frac{d \Omega}{d r} d r+\frac{d \Omega}{d v} d v+\frac{d \Omega}{d y} d y$.

I have given the complete expression, as it may be useful; but for the present purpose it will be sufficient to attend to the terms involving $\Theta, \Sigma, \Phi$. We have

$$
\begin{aligned}
& \frac{d \Omega}{d \Theta} d \Theta+\frac{d \Omega}{d \Sigma} d \Sigma+\frac{d \Omega}{d \Phi} d \Phi \\
& =\sec y \frac{d \Omega}{d v}\left\{\begin{array}{l}
-\sin y[\sin (v-\Sigma) \sin \Phi d \Theta+\cos (v-\Sigma) d \Phi] \\
+\cos y[-d \Sigma+\cos \Phi d \Theta
\end{array}\right\} \\
& -\frac{d \Omega}{d y}\{\cos (v-\Sigma) \sin \Phi d \Theta-\sin (v-\Sigma) d \Phi \quad\} \text {. }
\end{aligned}
$$

and consequently 


$$
\begin{aligned}
& \frac{d \Omega}{d \Sigma}=\quad-\frac{d \Omega}{d v}, \\
& \frac{d \Omega}{d \Theta}=\{\cos \Phi-\tan y \sin (v-\Sigma) \sin \Phi\} \frac{d \Omega}{d v}-\cos (v-\Sigma) \sin \Phi \frac{d \Omega}{d y}, \\
& \frac{d \Omega}{d \Phi}=\quad-\tan y \cos (v-\Sigma) \frac{d \Omega}{d v}+\sin (v-\Sigma) \quad \frac{d \Omega}{d y} ;
\end{aligned}
$$

which, it is to be observed, are partial differential equations satisfied by $\Omega$ considered as a function of the form $\Omega=\Omega(r, v, y, \Theta, \Sigma, \Phi) ; \Omega$ is, in fact, a function of three quantities only (say the coordinates $x_{0}, y_{0}, z_{0}$ ); and it is clear, $\grave{a}$ priori, that when thus expressed as a function of six quantities, it must satisfy three partial differential equations.

I write in the second equation $-\frac{d \Omega}{d \Sigma}$ for $\frac{d \Omega}{d v}$, and I then put $y=0$; we thus have

$$
\begin{array}{ll}
\cos (v-\Sigma) \frac{d \Omega}{d y}=-\cot \Phi \frac{d \Omega}{d \Sigma}-\operatorname{cosec} \Phi \frac{d \Omega}{d \Theta}, \\
\sin (v-\Sigma) \frac{d \Omega}{d y}= & \frac{d \Omega}{d \Phi} .
\end{array}
$$

I propose, as already mentioned, to apply the formulæ to the demonstration of the results obtained in my Memoir on the Problem of Disturbed Elliptic Motion. It will be remembered that $x_{0} y_{0}$ and $x_{0}$ denote a fixed orbit and fixed origin therein, $x_{1} y_{1}$ and $x_{1}$ an arbitrarily varying orbit and origin therein. I assume, however, that $x_{1}$ is a departure-point, so that we have $-d \sigma^{\prime}+\cos \phi^{\prime} d \theta^{\prime}=0$. The orbit $x_{2} y_{2}$ will be taken to be the varying instantaneous orbit of the body itself, that is, we have constantly $y=0$; and it is assumed that $x_{2}$ is a departure-point in this orbit. To conform to my former notation, I write $p$ instead of $v$; the position of the body in the varying instantaneous orbit is consequently determined by

$$
\begin{aligned}
& r \text {, the radius vector, } \\
& p \text {, the departure. }
\end{aligned}
$$

The entire displacement of the body arises from the displacements $d r$ and $r d p$ in the direction of the radius vector, and perpendicular to this direction, in the instantaneous orbit; that is, we have $Q=0, R=d p$, and the expression for the Vis viva function is simply

$$
T d t^{2}=\frac{1}{2}\left(d r^{2}+r^{2} d p^{2}\right)
$$

and, putting in the foregoing expressions of $Q$ and $R, y=0$, and $p$ in the place of $v$, the equations $Q=0, R d t=d p$, give

$$
\begin{aligned}
\cos (p-\Sigma) \sin \phi d \Theta-\sin (p-\Sigma) d \Phi= & \\
& \sin (p-\Sigma) \quad\left[\sin \left(\Theta-\sigma^{\prime}\right) \sin \phi^{\prime} d \theta^{\prime}+\cos \left(\Theta-\sigma^{\prime}\right) d \phi^{\prime}\right] \\
- & \cos (p-\Sigma) \cos \Phi\left[\cos \left(\Theta-\sigma^{\prime}\right) \sin \phi^{\prime} d \theta^{\prime}-\sin \left(\Theta-\sigma^{\prime}\right) d \phi^{\prime}\right],
\end{aligned}
$$

$d \Sigma-\cos \Phi d \Theta=$

$$
-\sin \Phi\left[\cos \left(\Theta-\sigma^{\prime}\right) \sin \phi^{\prime} d \theta^{\prime}-\sin \left(\Theta-\sigma^{\prime}\right) d \phi^{\prime}\right] .
$$


Now considering $r, p, y$ as the coordinates ( $y$ being ultimately put equal to zero) the general equations of motion are

$$
\begin{aligned}
& \frac{d}{d t}\left(\frac{d T}{d \dot{r}}\right)-\frac{d T}{d r}=\frac{d V}{d r}, \\
& \frac{d}{d t}\left(\frac{d T}{d \dot{p}}\right)-\frac{d T}{d p}=\frac{d V}{d p}, \\
& \frac{d}{d t}\left(\frac{d T}{d \dot{y}}\right)-\frac{d T}{d y}=\frac{d V}{d y}
\end{aligned}
$$

if for the moment $\dot{r}, \dot{p}, \dot{y}$ denote the differential coefficients of $r, p, y$ : the expression of the force function $V$ is $V=-\frac{n^{2} a^{3}}{r}+\Omega$ where $n^{2} a^{3}$ denotes an absolute constant, the sum of the masses, and the disturbing function $\Omega$ has a contrary sign to that of the Mécanique Céleste. We may in the first and second equations write at once $T d t^{2}=\frac{1}{2}\left(d r^{2}+r^{2} d p^{2}\right)$, and these equations thus become

$$
\begin{aligned}
& \frac{d}{d t} \quad \frac{d r}{d t}-r\left(\frac{d p}{d t}\right)^{2}=\frac{n^{2} a^{3}}{r^{2}}+\frac{d \Omega}{d r}, \\
& \frac{d}{d t}\left(r^{2} \frac{d p}{d t}\right)=\frac{d \Omega}{d p} .
\end{aligned}
$$

If in the third equation we take the general value of $T$ and after the differentiation with respect to $\dot{y}$, make the substitutions $y=0, Q=0, R d t=d p$, we find

$$
\frac{d T}{d \dot{y}}=0
$$

and the equation thus becomes

$$
-\frac{d T}{d y}=\frac{d \Omega}{d y}
$$

But $T d t^{2}=\frac{1}{2}\left\{d r^{2}+r^{2}\left(Q^{2}+R^{2}\right) d t^{2}\right\}$, consequently

$$
-\frac{d \Omega}{d y} d t=\frac{d T}{d y} d t=r^{2}\left(Q \frac{d Q}{d y}+R \frac{d R}{d y}\right) d t=r^{2} d p \frac{d R}{d y},
$$

and from the value of $R$, putting after the differentiation $y=0$, we find

$$
\begin{aligned}
& \frac{d R}{d y} d t=- \\
& {[\sin (p-\Sigma) \sin \Phi d \Theta+\cos (p-\Sigma) d \Phi]} \\
& -\cos (p-\Sigma) \quad\left[\sin \left(\Theta-\sigma^{\prime}\right) \sin \phi^{\prime} d \theta^{\prime}+\cos \left(\Theta-\sigma^{\prime}\right) d \phi^{\prime}\right] \\
& -\sin (p-\Sigma) \cos \Phi\left[\cos \left(\Theta-\sigma^{\prime}\right) \sin \phi^{\prime} d \theta-\sin \left(\Theta-\sigma^{\prime}\right) d \phi^{\prime}\right] .
\end{aligned}
$$


I write $r^{2} \frac{d p}{d t}=h$. We thus have

and

$$
\begin{aligned}
\frac{d}{d t} \frac{d r}{d t}-r\left(\frac{d p}{d t}\right)^{2} & =\frac{n^{2} a^{3}}{r^{2}}+\frac{d \Omega}{d r}, \\
r^{2} \frac{d p}{d t} & =h
\end{aligned}
$$

the remaining equations are

$$
d h=\frac{d \Omega}{d p} d t
$$

$$
\begin{aligned}
d \Sigma-\cos \Phi d \Theta & =\quad-\sin \Phi\left[\cos \left(\Theta-\sigma^{\prime}\right) \sin \phi^{\prime} d \theta^{\prime}-\sin \left(\Theta-\sigma^{\prime}\right) d \phi^{\prime}\right] \\
\cos (p-\Sigma) \sin \Phi d \Theta-\sin (p-\Sigma) d \Phi= & \sin (p-\Sigma) \quad\left[\sin \left(\Theta-\sigma^{\prime}\right) \sin \phi^{\prime} d \theta^{\prime}+\cos \left(\Theta-\sigma^{\prime}\right) d \phi^{\prime}\right] \\
- & \cos (p-\Sigma) \cos \Phi\left[\cos \left(\Theta-\sigma^{\prime}\right) \sin \phi^{\prime} d \theta^{\prime}-\sin \left(\Theta-\sigma^{\prime}\right) d \phi^{\prime}\right]
\end{aligned}
$$

$\sin (p-\Sigma) \sin \Phi d \Theta+\cos (p-\Sigma) d \Phi=$

$$
\begin{aligned}
& \frac{1}{h} \frac{d \Omega}{d y} d t \\
& -\cos (\beta-\Sigma) \quad\left[\sin \left(\Theta-\sigma^{\prime}\right) \sin \phi^{\prime} d \theta^{\prime}+\cos \left(\Theta-\sigma^{\prime}\right) d \phi^{\prime}\right] \\
& -\sin (p-\Sigma) \cos \Phi\left[\cos \left(\Theta-\sigma^{\prime}\right) \sin \phi^{\prime} d \theta^{\prime}-\sin \left(\Theta-\sigma^{\prime}\right) d \phi^{\prime}\right] .
\end{aligned}
$$

Moreover we have

$$
\begin{aligned}
& \cos (p-\Sigma) \frac{d \Omega}{d y}=-\cot \phi \frac{d \Omega}{d \Sigma}-\operatorname{cosec} \Phi \frac{d \Omega}{d \Theta}, \\
& \sin (p-\Sigma) \frac{d \Omega}{d y}=\quad \frac{d \Omega}{d \Phi},
\end{aligned}
$$

by means of which the preceding two equations are reduced to

$$
\begin{aligned}
d \Theta= & \frac{\operatorname{cosec} \Phi}{h} \frac{d \Omega}{d \Phi} d t \\
& -\cot \Phi\left[\cos \left(\Theta-\sigma^{\prime}\right) \sin \phi^{\prime} d \theta^{\prime}-\sin \left(\Theta-\sigma^{\prime}\right) d \phi^{\prime}\right] \\
d \Phi= & -\frac{\cot \Phi}{h} \frac{d \Omega}{d \Sigma} d t-\frac{\operatorname{cosec} \Phi}{h} \frac{d \Omega}{d \Theta} d t \\
& -\quad\left[\sin \left(\Theta-\sigma^{\prime}\right) \sin \phi^{\prime} d \theta^{\prime}+\cos \left(\Theta-\sigma^{\prime}\right) d \phi^{\prime}\right]
\end{aligned}
$$

and we then have

$$
\begin{aligned}
d \Sigma= & \frac{\cot \Phi}{h} \frac{d \Omega}{d \Phi} d t \\
& -\operatorname{cosec} \Phi\left[\cos \left(\Theta-\sigma^{\prime}\right) \sin \phi^{\prime} d \theta^{\prime}-\sin \left(\Theta-\sigma^{\prime}\right) d \phi^{\prime}\right]
\end{aligned}
$$


The entire system consequently is

and

$$
\begin{aligned}
& \frac{d}{d t}\left(\frac{d r}{d t}\right)-r\left(\frac{d p}{d t}\right)^{2}=\frac{n^{2} a^{3}}{r^{2}}+\frac{d \Omega}{d r} \\
& r^{2} \frac{d p}{d t}=h
\end{aligned}
$$

$$
\begin{array}{ll}
d h= & \frac{d \Omega}{d p} d t, \\
d \Theta= & \frac{\operatorname{cosec} \Phi}{h} \frac{d \Omega}{d \Phi} d t \quad-\cot \Phi\left[\cos \left(\Theta-\sigma^{\prime}\right) \sin \phi^{\prime} d \theta^{\prime}-\sin \left(\Theta-\sigma^{\prime}\right) d \phi^{\prime}\right] \\
d \Sigma= & \frac{\cot \Phi}{h} \frac{d \Omega}{d \Phi} d t \quad-\operatorname{cosec} \Phi\left[\cos \left(\Theta-\sigma^{\prime}\right) \sin \phi^{\prime} d \theta^{\prime}-\sin \left(\Theta-\sigma^{\prime}\right) d \phi^{\prime}\right], \\
d \Phi=-\frac{\operatorname{cosec} \Phi}{h} \frac{d \Omega}{d \Theta} d t-\frac{\cot \Phi}{h} \frac{d \Omega}{d \Sigma} d t-\left[\sin \left(\Theta-\sigma^{\prime}\right) \sin \phi^{\prime} d \theta^{\prime}+\cos \left(\Theta-\sigma^{\prime}\right) d \phi^{\prime}\right]
\end{array}
$$

where $\Omega=\Omega(r, p, \Theta, \Sigma, \Phi)$. We may add the before-mentioned equation,

$$
d \Sigma-\cos \Phi d \Theta=\quad-\sin \Phi\left[\cos \left(\Theta-\sigma^{\prime}\right) \sin \phi^{\prime} d \theta^{\prime}-\sin \left(\Theta-\sigma^{\prime}\right) d \phi^{\prime}\right] .
$$

To obtain the formulæ of my Memoir before referred to, it would only be necessary to write $h=n a^{2} \sqrt{1-e^{2}}$, and complete the solution by applying to the first equation the method of the variation of the elements; but I prefer leaving the system in its present form, as it is one which may perhaps be useful.

The formulæ have especial reference to the lunar theory, and the orbit $x_{0} y_{0}$ denotes the fixed ecliptic, $x_{1} y_{1}$ the varying ecliptic, and $x_{2} y_{2}$ the instantaneous orbit of the moon. But if, instead of considering with Hansen the instantaneous orbit of the moon, the position of the moon is determined (as in the theories of Clairaut, Plana, and others) by

$$
\begin{aligned}
& r \text {, the radius vector, } \\
& v \text {, the longitude, } \\
& y \text {, the latitude, }
\end{aligned}
$$

where the longitude is measured on the variable ecliptic, then if, in the general expressions for $P, Q, R$, we first neglect the terms involving $d \theta^{\prime}, d \sigma^{\prime}, d \phi^{\prime}$, and then write $\theta^{\prime}, \sigma^{\prime}, \phi^{\prime}$, in the place of $\Theta, \Sigma, \Phi$, we find

$$
\begin{aligned}
P d t= & \sin y d v \\
& +\cos y\left[\sin \left(v-\sigma^{\prime}\right) \sin \phi^{\prime} d \theta^{\prime}+\cos \left(v-\sigma^{\prime}\right) d \phi^{\prime}\right] \\
& +\sin y\left[-d \sigma^{\prime}+\cos \phi^{\prime} d \theta^{\prime}\right] \\
Q d t= & -d y \\
& +\quad\left[\cos \left(v-\sigma^{\prime}\right) \sin \phi^{\prime} d \theta^{\prime}-\sin \left(v-\sigma^{\prime}\right) d \phi^{\prime}\right]
\end{aligned}
$$




$$
\begin{aligned}
R d t= & \cos y d v \\
& -\sin y\left[\sin \left(v-\sigma^{\prime}\right) \sin \phi^{\prime} d \theta^{\prime}+\cos \left(v-\sigma^{\prime}\right) d \phi^{\prime}\right] \\
& +\cos y\left[-d \sigma^{\prime}+\cos \phi^{\prime} d \theta^{\prime}\right.
\end{aligned}
$$

and with these values the expression for the Vis viva function is, as before,

$$
T d t^{2}=\frac{1}{2}\left\{d r^{2}+r^{2}\left(Q^{2}+R^{2}\right) d t^{2}\right\} .
$$

The longitude may be measured from a departure-point, and then the expressions for $P, Q, R$, may be simplified by omitting the terms which contain $-d \sigma^{\prime}+\cos \phi^{\prime} d \theta^{\prime}$.

\section{Addition.}

I have in the course of the memoir used some properties connected with the theory of rotation, but it is proper to give the analytical investigation.

Suppose that the rectangular coordinates $(X, Y, Z)$ are connected with the rectangular coordinates $\left(X, Y_{1}, Z_{1}\right)$ by the equations

then we have

$$
\begin{aligned}
& X=\alpha X_{1}+\beta Y_{1}+\gamma Z_{\prime}, \\
& Y=\alpha^{\prime} X_{1}+\beta^{\prime} Y_{1}+\gamma^{\prime} Z_{\prime}, \\
& Z=\alpha^{\prime \prime} X_{1}+\beta^{\prime \prime} Y_{1}+\gamma^{\prime \prime} Z_{\prime}
\end{aligned}
$$

$$
\begin{aligned}
& d X=\alpha d X,+\beta d Y,+\gamma d Z,+X, d \alpha+Y, d \beta+Z, d \gamma, \\
& d Y=\alpha^{\prime} d X,+\beta^{\prime} d Y,+\gamma^{\prime} d Z,+X, d \alpha^{\prime}+Y, d \beta^{\prime}+Z, d \gamma^{\prime}, \\
& d Z=\alpha^{\prime \prime} d X,+\beta^{\prime \prime} d Y,+\gamma^{\prime \prime} d Z,+X, d \alpha^{\prime \prime}+Y, d \beta^{\prime \prime}+Z, d \gamma^{\prime \prime},
\end{aligned}
$$

and then, putting

$$
\begin{aligned}
& \mathrm{p} d t=\gamma d \beta+\gamma^{\prime} d \beta^{\prime}+\gamma^{\prime \prime} d \beta^{\prime \prime}, \\
& \mathrm{q} d t=\alpha d \gamma+\alpha^{\prime} d \gamma^{\prime}+\alpha^{\prime \prime} d \gamma^{\prime \prime}, \\
& \mathrm{r} d t=\beta d \alpha+\beta^{\prime} d \alpha^{\prime}+\beta^{\prime \prime} d \alpha^{\prime \prime},
\end{aligned}
$$

where $\mathrm{p}, \mathrm{q}, \mathrm{r}$ are the rotations round $X_{1}, Y_{1}, Z_{\imath}$, from $Y$, to $Z, Z, Z$, to $X_{1}$, and $X$, to $Y$, respectively, we have

$$
\begin{aligned}
& \alpha d X+\alpha^{\prime} d Y+\alpha^{\prime \prime} d Z=d X_{1}+\left(-\mathrm{r} Y_{1}+\mathrm{q} Z_{l}\right) d t, \\
& \beta d X+\beta^{\prime} d Y+\beta^{\prime \prime} d Z=d Y_{1}+\left(-\mathrm{p} Z_{1}+\mathrm{r} X_{l}\right) d t, \\
& \gamma d X+\gamma^{\prime} d Y+\gamma^{\prime \prime} d Z=d Z_{1}+\left(-\mathrm{q} X_{1}+\mathrm{p} Y_{l}\right) d t,
\end{aligned}
$$

where, considering $X Y Z$ as fixed axes, the left-hand sides are the displacements in the directions of the moveable axes $X, Y, Z$, arising from the variations of the coordinates $X, Y, Z$, and the variation of the position of these axes; and these displace-

C. III. 
ments have therefore the values given by the right-hand sides. This is, of course, well known. It may be added that if the axes $X Y Z$ are moveable, and $d X, d Y, d Z$ denote the displacements in the directions of these axes, the formulæ are still true.

Suppose that $\Omega$ is a function of $X, Y, Z$; say $\Omega=\Omega(X, Y, Z)$, we have

$$
d \Omega=\frac{d \Omega}{d X} d X+\frac{d \Omega}{d Y} d Y+\frac{d \Omega}{d Z} d Z
$$

which may be written

$$
\begin{aligned}
d \Omega= & \left(\alpha \frac{d \Omega}{d X}+\alpha^{\prime} \frac{d \Omega}{d Y}+\alpha^{\prime \prime} \frac{d \Omega}{d Z}\right)\left(\alpha d X+\alpha^{\prime} d Y+\alpha^{\prime \prime} d Z\right) \\
& +\left(\beta \frac{d \Omega}{d X}+\beta^{\prime} \frac{d \Omega}{d Y}+\beta^{\prime \prime} \frac{d \Omega}{d Z}\right)\left(\beta d X+\beta^{\prime} d Y+\beta^{\prime \prime} d Z\right) \\
& +\left(\gamma \frac{d \Omega}{d X}+\gamma^{\prime} \frac{d \Omega}{d Y}+\gamma^{\prime \prime} \frac{d \Omega}{d Z}\right)\left(\gamma d X+\gamma^{\prime} d Y+\gamma^{\prime \prime} d Z\right)
\end{aligned}
$$

. and the coefficients of transformation $\alpha, \beta, \gamma$, \&c., being independent of $X, Y, Z$ and $X_{1}, Y_{1}, Z_{1}$, the first factors are $\frac{d \Omega}{d X}, \frac{d \Omega}{d Y,}, \frac{d \Omega}{d Z}$, and we have

$$
\begin{aligned}
d \Omega= & \frac{d \Omega}{d X_{1}}\left[d X_{1}+\left(-\mathrm{r} Y_{1}+\mathrm{q} Z_{l}\right) d t\right] \\
& +\frac{d \Omega}{d Y_{1}}\left[d Y_{1}+\left(-\mathrm{p} Z_{+}+\mathrm{r} X_{\imath}\right) d t\right] \\
& +\frac{d \Omega}{d Z}\left[d Z_{1}+\left(-\mathrm{q} X+\mathrm{p} Y_{1}\right) d t\right]
\end{aligned}
$$

which is a theorem assumed in the memoir.

The expressions of $P, Q, R$ in the memoir depend upon the composition of several rotations. If, in Lamé's very convenient notation, we write

\begin{tabular}{c|c|c|c|} 
& $X_{\cdot}$ & $Y_{,}$ & $Z_{,}$ \\
\hline$X$ & $a$ & $\beta$ & $\gamma$ \\
\hline$Y$ & $a^{\prime}$ & $\beta^{\prime}$ & $\gamma^{\prime}$ \\
\hline$Z$ & $\alpha^{\prime \prime}$ & $\beta^{\prime \prime}$ & $\gamma^{\prime \prime}$ \\
\hline & & \\
\hline www.rcin.org.pl
\end{tabular}


to denote the before-mentioned relation between $(X, Y, Z)$ and $\left(X_{\imath}, Y_{l}, Z_{\imath}\right)$ then the tables

\begin{tabular}{c|c|c|c|} 
& $X_{\prime}$, & $Y_{,}$ & $Z_{\prime}$ \\
\hline$X$ & $\alpha$ & $\beta$ & $\gamma$ \\
\hline$Y$ & $\alpha^{\prime}$ & $\beta^{\prime}$ & $\gamma^{\prime}$ \\
\hline$Z$ & $a^{\prime \prime}$ & $\beta^{\prime \prime}$ & $\gamma^{\prime \prime}$ \\
\hline
\end{tabular}

\begin{tabular}{c|c|c|c|} 
& $X_{2}$ & $Y_{2}$ & $Z_{2}$ \\
\hline$X_{1}$ & $A$ & $B$ & $C$ \\
\hline$Y_{,}$ & $A^{\prime}$ & $B^{\prime}$ & $C^{\prime}$ \\
\hline$Z_{\prime}$ & $A^{\prime \prime}$ & $B^{\prime \prime}$ & $C^{\prime \prime}$ \\
\hline
\end{tabular}

lead to the table

\begin{tabular}{|c|c|c|}
\hline$X$ & $Y_{2}$ & $Z_{2}$ \\
\hline$(\alpha, \beta, \gamma)\left(A, A^{\prime}, A^{\prime \prime}\right)$ & $(\alpha, \beta, \gamma)\left(B, B^{\prime}, B^{\prime \prime}\right)$ & $(\alpha, \beta, \gamma)\left(C,: C^{\prime \prime}, C^{\prime \prime}\right)$ \\
\hline$\left(\alpha^{\prime}, \beta^{\prime}, \gamma^{\prime}\right)\left(A, A^{\prime}, A^{\prime \prime}\right)$ & $\left(\alpha^{\prime}, \beta^{\prime}, \gamma^{\prime}\right)\left(B, B^{\prime}, B^{\prime \prime}\right)$ & $\left(\alpha^{\prime}, \beta^{\prime}, \gamma^{\prime}\right)\left(C, C^{\prime}, C^{\prime \prime}\right)$ \\
\hline$\left(\alpha^{\prime \prime}, \beta^{\prime \prime}, \gamma^{\prime \prime}\right)\left(A, A^{\prime}, A^{\prime \prime}\right)$ & $\left(a^{\prime \prime}, \beta^{\prime \prime}, \gamma^{\prime \prime}\right)\left(B, B^{\prime}, B^{\prime \prime}\right)$ & $\left(\alpha^{\prime \prime}, \beta^{\prime \prime}, \gamma^{\prime \prime}\right)\left(C, C^{\prime}, C^{\prime \prime}\right)$ \\
\hline
\end{tabular}

where for shortness $(\alpha, \beta, \gamma)\left(A, A^{\prime}, A^{\prime \prime}\right)$, \&c., stand for $\alpha A+\beta A^{\prime}+\gamma A^{\prime \prime}$, \&c. these are of course the ordinary formulæ for the composition of transformations from one set of rectangular axes to another. We may say that the coefficients of transformation from $(X, Y, Z)$ to $\left(X, Y_{1}, Z_{l}\right)$ are $(\alpha, \beta, \gamma, \& c$.$) . And in dealing with several sets of$ coordinates, the coefficients of transformation may be distinguished by suffixes. Thus I assume that the coefficients of transformation

$$
\begin{aligned}
& \text { from }\left(x_{0}, y_{0}, z_{0}\right) \text { to }\left(x_{x}, y_{1}, z_{1}\right) \text { are }(\alpha, \beta, \gamma, \& c .)_{01} \text {, } \\
& \text { ” }\left(x_{1}, y_{1}, z_{1}\right) \text { \#( }\left(x_{2}, y_{2}, z_{2}\right) \text {, }(\alpha, \beta, \gamma, \& \mathrm{c})_{12} \text {, } \\
& \text { ” }\left(x_{0}, y_{0}, z_{0}\right),\left(x_{2}, y_{2}, z_{2}\right) \text { \# }(\alpha, \beta, \gamma, \& c .)_{02} \text {, }
\end{aligned}
$$

where $(\alpha, \beta, \gamma, \& c .)_{02}$ can be obtained as above from $(\alpha, \beta, \gamma, \& c .)_{01}$ and $(\alpha, \beta, \gamma, \& c .)_{12}$ The rotations $p, q, r$ may be distinguished by suffixes in like manner, viz., $(p, q, r)_{01}$ will denote the rotations from $\left(x_{0}, y_{0}, z_{0}\right)$ to $\left(x_{1}, y_{1}, z_{1}\right)$ and so on.

There is no difficulty in obtaining first

$$
\begin{aligned}
& p_{02}=p_{12}+\left(\alpha, \alpha^{\prime}, \alpha^{\prime \prime}\right)_{12}(p, q, r)_{01}, \\
& q_{02}=q_{12}+\left(\beta, \beta^{\prime}, \beta^{\prime \prime}\right)_{12}(p, q, r)_{01}, \\
& r_{02}=r_{12}+\left(\gamma, \gamma^{\prime}, \gamma^{\prime \prime}\right)_{12}(p, q, r)_{01},
\end{aligned}
$$


and then by a repetition of the like transformation

$$
\begin{aligned}
& p_{03}=p_{23}+\left(\alpha, \alpha^{\prime}, \alpha^{\prime \prime}\right)_{23}(p, q, r)_{12}+\left(\alpha, \alpha^{\prime}, \alpha^{\prime \prime}\right)_{13}(p, q, r)_{01}, \\
& q_{03}=q_{23}+\left(\beta, \beta^{\prime}, \beta^{\prime \prime}\right)_{23}(p, q, r)_{12}+\left(\beta, \beta^{\prime}, \beta^{\prime \prime}\right)_{13}(p, q, r)_{01}, \\
& r_{03}=r_{23}+\left(\gamma, \gamma^{\prime}, \gamma^{\prime \prime}\right)_{23}(p, q, r)_{12}+\left(\gamma, \gamma^{\prime}, \gamma^{\prime \prime}\right)_{13}(p, q, r)_{01},
\end{aligned}
$$

and in like manner for any number of systems, but for the present purpose this is enough, as $p_{03}, q_{03}, r_{03}$ are in fact the $P, Q, R$ of the memoir. And if $\theta^{\prime}, \sigma^{\prime}, \phi^{\prime}$, $\Theta, \Sigma, \Phi, v, y$ signify as before, then the coefficients of transformation from $\left(x_{0}, y_{0}, z_{0}\right)$ to $\left(x_{1}, y_{1}, z_{1}\right)$ are given by the table

\begin{tabular}{|c|c|c|}
\hline$x_{0}$ & $x_{1}$ & $y_{1}$ \\
\hline$y_{0} \cos \sigma^{\prime} \cos \theta^{\prime}+\sin \sigma^{\prime} \sin \theta^{\prime} \cos \phi^{\prime}$ & $\sin \sigma^{\prime} \cos \theta^{\prime}-\cos \sigma^{\prime} \sin \theta^{\prime} \cos \phi^{\prime}$ & $\sin \theta^{\prime} \sin \phi^{\prime}$ \\
\hline $\cos \sigma^{\prime} \sin \theta^{\prime}-\sin \sigma^{\prime} \cos \theta^{\prime} \cos \phi^{\prime}$ & $\sin \sigma^{\prime} \sin \theta^{\prime}+\cos \sigma^{\prime} \cos \theta^{\prime} \cos \phi^{\prime}$ & $-\cos \theta^{\prime} \sin \phi^{\prime}$ \\
\hline$z_{0}$ & $\cos \sigma^{\prime} \sin \phi^{\prime}$ & $\cos \phi^{\prime}$ \\
\hline
\end{tabular}

and the corresponding rotation coefficients are

\begin{tabular}{|c|c|c|}
\hline $\cos \Sigma \cos \Theta+\sin \Sigma \sin \Theta \cos \Phi$ & $\sin \Sigma \cos \Theta-\cos \Sigma \sin \Theta \cos \Phi$ & $\sin \Theta \sin \Phi$ \\
\hline $\cos \Sigma \sin \Theta-\sin \Sigma \cos \Theta \cos \Phi$ & $\sin \Sigma \sin \Theta+\cos \Sigma \cos \Theta \cos \Phi$ & $-\cos \Theta \sin \Phi$ \\
\hline$-\sin \Sigma \sin \Phi$ & $\cos \Sigma \sin \Phi$ & $\cos \Phi$ \\
\hline
\end{tabular}

$$
\begin{aligned}
& \mathrm{p}_{01} d t=-\sin \sigma^{\prime} \sin \phi^{\prime} d \theta^{\prime}+\cos \sigma^{\prime} d \phi^{\prime}, \\
& \mathrm{q}_{01} d t=\cos \sigma^{\prime} \sin \phi^{\prime} d \theta^{\prime}+\sin \sigma^{\prime} d \phi^{\prime}, \\
& \mathrm{r}_{01} d t=-d \sigma^{\prime}+\cos \phi^{\prime} d \theta^{\prime} .
\end{aligned}
$$

The coefficients of transformation from $\left(x_{1}, y_{1}, z_{1}\right)$ to $\left(x_{2}, y_{2}, z_{2}\right)$ are given by the table

and the corresponding rotation coefficients are

$$
\begin{aligned}
& \mathrm{p}_{12} d t=-\sin \Sigma \sin \Phi d \Theta+\cos \Sigma d \Phi, \\
& \mathrm{q}_{12} d t=+\cos \Sigma \sin \Phi d \Theta+\sin \Sigma d \Phi, \\
& \mathrm{r}_{12} d t=-d \Sigma+\cos \Phi d \Theta,
\end{aligned}
$$


and the coefficients of transformation from $\left(x_{2}, y_{2}, z_{2}\right)$ to $\left(x_{3}, y_{3}, z_{3}\right)$ are given by the table

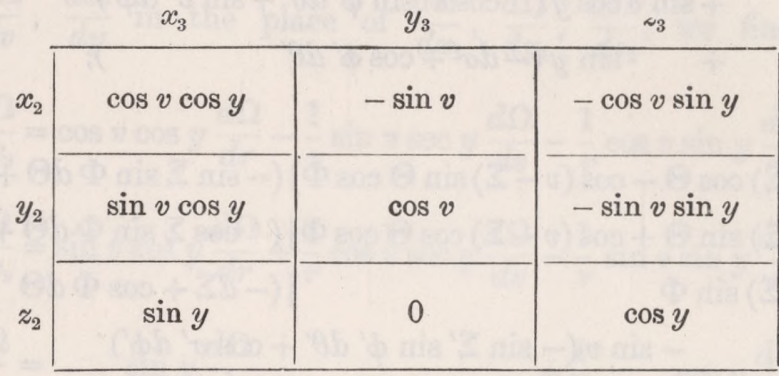

and the corresponding rotation coefficients are given by

$$
\begin{aligned}
& \mathrm{p}_{23} d t=-\sin y d v, \\
& \mathrm{q}_{23} d t=-d y, \\
& \mathrm{r}_{23} d t=\cos y d v .
\end{aligned}
$$

From the last two tables it is easy to deduce the coefficients of transformation from $\left(x_{1}, y_{1}, z_{1}\right)$ to $\left(x_{3}, y_{3}, z_{3}\right)$, these are given by the table

$y_{3}$

$\cos y\{\cos (v-\Sigma) \cos \Theta-\sin (v-\Sigma) \sin \Theta \cos \Phi\}+\sin y \sin \Theta \sin \Phi$ $-\sin (v-\Sigma) \cos \Theta-\cos (v-\Sigma) \sin \Theta \cos \Phi$

$\cos y\{\cos (v-\Sigma) \sin \Theta+\sin (v-\Sigma) \cos \Theta \cos \Phi\}-\sin y \cos \Theta \sin \Phi$ $-\sin (v-\Sigma) \sin \Theta+\cos (v-\Sigma) \cos \Theta \cos \Phi$

$\cos y\{\sin (v-\Sigma) \sin \Phi$ \}$+\sin y \cos \Phi$ $\cos (v-\Sigma) \sin \Phi$

$-\sin y\{\cos (v-\Sigma) \cos \Theta-\sin (v-\Sigma) \sin \Theta \cos \Phi\}+\cos y \sin \Theta \sin \Phi$

$-\sin y\{\cos (v-\Sigma) \sin \Theta+\sin (v-\Sigma) \cos \Theta \cos \Phi\}-\cos y \cos \Theta \sin \Phi$

$-\sin y\{\sin (v-\Sigma) \sin \Phi$ \}$+\cos y \cos \Phi$

It is now easy to form the values of $P, Q, R$. We have

$P d t=\sin y d v$

$+[\cos y\{\cos (v-\Sigma) \cos \Theta-\sin (v-\Sigma) \sin \Theta \cos \Phi\}+\sin y \sin \Theta \sin \Phi](-\sin \Sigma \sin \Phi d \Theta+\cos \Sigma d \Phi)$

$+[\cos y\{\cos (v-\Sigma) \sin \Theta+\sin (v-\Sigma) \cos \Theta \cos \Phi\}-\sin y \cos \Theta \sin \Sigma](\quad \cos \Sigma \sin \Phi d \Theta+\sin \Sigma d \Phi)$

$+[\cos y\{\sin (v-\Sigma) \sin \Phi$

\}$+\sin y \cos \Phi \quad](-d \Sigma+\cos \Phi d \Theta$ 


$$
\begin{aligned}
& +\cos v \cos y\left(-\sin \sigma^{\prime} \sin \phi^{\prime} d \theta^{\prime}+\cos \sigma^{\prime} d \phi^{\prime}\right) \\
& +\sin v \cos y\left(\cos \sigma^{\prime} \sin \phi^{\prime} d \theta^{\prime}+\sin \sigma^{\prime} d \phi^{\prime}\right) \\
& +\quad \sin y\left(-d \sigma^{\prime}+\cos \phi^{\prime} d \theta^{\prime}\right.
\end{aligned}
$$

$Q d t=-d y$

$+[-\sin (v-\Sigma) \cos \Theta-\cos (v-\Sigma) \sin \Theta \cos \Phi](-\sin \Sigma \sin \Phi d \Theta+\cos \Sigma d \Phi)$

$+[-\sin (v-\Sigma) \sin \Theta+\cos (v-\Sigma) \cos \Theta \cos \Phi](\cos \Sigma \sin \Phi d \Theta+\sin \Sigma d \Phi)$

$+[\cos (v-\Sigma) \sin \Phi \quad](-d \Sigma+\cos \Phi d \Theta$

$$
\begin{aligned}
& -\sin v\left(-\sin \Sigma^{\prime} \sin \phi^{\prime} d \theta^{\prime}+\cos \sigma^{\prime} d \phi^{\prime}\right) \\
& +\cos v\left(\cos \Sigma^{\prime} \sin \phi^{\prime} d \theta^{\prime}+\sin \sigma^{\prime} d \phi^{\prime}\right) \\
& +0\left(-d \Sigma^{\prime}+\cos \phi^{\prime} d \theta^{\prime}\right),
\end{aligned}
$$

$R d t=\cos y d v$

$+[-\sin y\{\cos (v-\Sigma) \cos \Theta-\sin (v-\Sigma) \sin \Theta \cos \Phi\}+\cos y \sin \Theta \sin \Phi](-\sin \Sigma \sin \Phi d \Theta+\cos \Sigma d \Phi)$

$+[-\sin y\{\cos (v-\Sigma) \sin \Theta+\sin (v-\Sigma) \cos \Theta \cos \Phi\}-\cos y \cos \Theta \sin \Phi](\cos \Sigma \sin \Phi d \Theta+\sin \Sigma d \Phi)$

$+[-\sin y\{\sin (v-\Sigma) \sin \Phi \quad\}+\cos y \cos \Phi \quad](-d \Sigma+\cos \Phi d \Theta$

$-\cos v \sin y\left(-\sin \sigma^{\prime} \sin \phi^{\prime} d \theta^{\prime}+\cos \sigma^{\prime} d \phi^{\prime}\right)$

$-\sin v \sin y\left(\cos \sigma^{\prime} \sin \phi^{\prime} d \theta^{\prime}+\sin \sigma^{\prime} d \phi^{\prime}\right)$

$+\cos y \quad\left(-d \sigma^{\prime}+\cos \phi^{\prime} d \theta^{\prime} \quad\right)$,

which are at once reducible into the before-mentioned form.

It only remains to prove the expressions for $\frac{d \Omega}{d x_{3}}, \frac{d \Omega}{d y_{3}}, \frac{d \Omega}{d z_{3}}$ assumed in the memoir. The relations between $\left(x_{3}, y_{3}, z_{3}\right)$ and $\left(x_{2}, y_{2}, z_{2}\right)$ give

$$
\begin{aligned}
& \frac{d \Omega}{d x_{3}}=\cos v \cos y \frac{d \Omega}{d x_{2}}+\sin v \cos y \frac{d \Omega}{d y_{2}}+\sin y \frac{d \Omega}{d z_{2}^{\prime}}, \\
& \frac{d \Omega}{d y_{3}}=-\sin v \frac{d \Omega}{d x_{2}}+\cos v \quad \frac{d \Omega}{d y_{2}}, \\
& \frac{d \Omega}{d z_{3}}=-\cos v \sin y \frac{d \Omega}{d x_{2}}-\sin v \sin y \frac{d \Omega}{d y_{2}}+\cos y \frac{d \Omega}{d z_{2}},
\end{aligned}
$$

these formulæ apply to any point whatever the coordinates of which to the one set of axes are $\left(x_{2}, y_{2}, z_{2}\right)$ and to the other set $\left(x_{3}, y_{3}, z_{3}\right)$ and in obtaining them the coefficients of transformation $v, y$ are of course considered as independent of either set of coordinates. But the coordinates $\left(x_{2}, y_{2}, z_{2}\right)$ of the body are

$x_{2}=r \cos y \cos v$,

$y_{2}=r \cos y \sin v$, 
and using the foregoing equations in order to introduce into the formulæ the differential coefficients $\frac{d \Omega}{d r}, \frac{d \Omega}{d v}, \frac{d \Omega}{d y}$ in the place of $\frac{d \Omega}{d x_{2}}, \frac{d \Omega}{d y_{2}}, \frac{d \Omega}{d z_{2}}$, we find

$$
\begin{aligned}
& \frac{d \Omega}{d x_{2}}=\cos v \cos y \frac{d \Omega}{d r}-\frac{1}{r} \sin v \sec y \frac{d \Omega}{d v}-\frac{1}{r} \cos v \sin y \frac{d \Omega}{d y}, \\
& \frac{d \Omega}{d y_{2}}=\sin v \cos y \frac{d \Omega}{d r}+\frac{1}{r} \cos v \sec y \frac{d \Omega}{d v}-\frac{1}{r} \sin v \sin y \frac{d \Omega}{d y}, \\
& \frac{d \Omega}{d z_{2}}=\sin y \frac{d \Omega}{d r} \quad+\frac{1}{r} \quad \cos y \frac{d \Omega}{d y} .
\end{aligned}
$$

Substituting these values we have the required formulæ

$$
\frac{d \Omega}{d x_{3}}=\frac{d \Omega}{d r}, \quad \frac{d \Omega}{d y_{3}}=\frac{1}{r} \sec y \frac{d \Omega}{d v}, \quad \frac{d \Omega}{d z_{3}}=\frac{1}{r} \frac{d \Omega}{d y}
$$

where on the right-hand side $\Omega=\Omega\left(r, v, y, \Theta, \Sigma, \Phi, \theta^{\prime}, \sigma^{\prime}, \phi^{\prime}\right)$.

2, Stone Buildings, W.C. 28th Dec., 1858. 